\title{
HAMMERSTEIN OPERATORS PRESERVING DISJOINTNESS
}

\author{
A. V. KOLDUNOV
}

(Communicated by Palle E. T. Jorgensen)

\begin{abstract}
This paper deals with the problems of a multiplicative representation and of automatic continuity of linear and nonlinear operators preserving disjointness. The operators satisfying a modified Hammerstein condition are introduced and investigated. In $\S 3$ we develop a theory of quasi-linear disjointness-preserving Hammerstein operators. As an application we prove that a bijective disjointness-preserving operator between Banach lattices is a continuous d-isomorphism, thus answering in the affirmative a problem posed by Y. Abramovich in 1992. We also construct an example demonstrating that the completeness of the "departure" space cannot be omitted in general.
\end{abstract}

\section{INTRODUCTION}

Recall that an operator $T: X \rightarrow Y$ between vector lattices is said to be disjointness preserving (or equivalently, $T$ preserves disjointness) if $|T u| \wedge|T v|=0$ in $Y$ whenever $|u| \wedge|v|=0$ in $X$.

The linear operators preserving disjointness have been studied extensively for the last decade (see, for instance, [A, AAK, AVK1,2, AB, Art, AH, Har, Kit1,2, HP1, W]). Two major directions of these investigations are the automatic continuity and the multiplicative representation of such operators. One of the purposes of this article is to show that the methods developed in [A, AVK1,2] for linear disjointness-preserving operators can be successfully modified and applied to some broad classes of nonlinear operators, in particular, to operators satisfying the Hammerstein property (see Definition 2.1). Not only is the resulting nonlinear theory of interest on its own, but it also has some important applications to the linear theory. One of these applications is given in Theorem 3.6 and Corollary 3.7, where we solve in the affirmative a problem posed by Y. Abramovich [HL] of whether the inverse of a one-to-one disjointness-preserving operator between Banach lattices is also disjointness preserving.

The remaining part of the introduction contains some notation and terminology which will be needed in what follows.

If $K$ is a compact (always Hausdorff) topological space and $f$ is a continuous function on $K$, then $\mathrm{cz}(f)$ denotes the cozero set of $f$, i.e., $\mathrm{cz}(f)=\{t \in K$ : $f(t) \neq 0\}$.

Received by the editors April 2, 1993 and, in revised form, June 28, 1993.

1991 Mathematics Subject Classification. Primary 46A40, 47H30.

Key words and phrases. Banach lattice, disjointness preserving operator. 
If $f$ and $g$ are two continuous functions on $K$ and $H$ is a subject of $K$, then, following [A], we write $f[H]=g$, provided $f(t)=g(t)$ for all $t$ in some open neighborhood $G(H)$ of the set $H$.

We assume throughout the work that all vector lattices are Archimedean. For each positive element $e$ of a vector lattice $X$ we denote by $X(e)$ the order ideal in $X$ generated by $e$, i.e., $X(e)=\{x \in X:(\exists \lambda \in \mathbb{R})(|x| \leq \lambda e)\}$.

Recall that each vector lattice may be represented (usually in many ways) as a vector lattice of extended continuous functions on some compact space $K_{X}$ (see [V] for details). As a rule, we do not distinguish between an element in $X$ and its representation. If some element $e \in X_{+}$is fixed, then we always can choose a representation of $X$ such that the image of $e$ (under this representation) is a characteristic function of some compact subset of $K_{X}$, and the elements from $X$ separate the points of this compact set, $\operatorname{supp}(e)=e^{-1}(1)$. We will denote such representations by $\{X, e\}$.

By a d-sequence we mean any sequence whose terms are pairwise disjoint. For terminology and notation not explained in the paper we refer to $[A B]$ or [V].

\section{OPERATORS SATISFYING THE HAMMERSTEIN PROPERTY}

We begin by recalling a known Hammerstein property.

Definition 2.1. Let $X$ and $Y$ be vector lattices, and let $T: X \rightarrow Y$ be a (not necessarily linear) operator such that $T 0=0$. We say that $T$ satisfies the Hammerstein property if for arbitrary disjoint $u, v \in X$ and any $x \in X$ the equality

$$
T(u+x+v)-T(u+x)=T(x+v)-T(x)
$$

holds.

Obviously each linear operator satisfies the Hammerstein property. Letting $x=0$ in the above equality and using that $T 0=0$, we see that $T(u+v)=$ $T(u)+T(v)$. That is, each $T$ with the Hammerstein property is disjointly additive. In the next definition we introduce a property which, at least formally, is much weaker than the Hammerstein property. However, as we shall see later on, both properties are often equivalent.

For the simplicity of the presentation we will assume from now on that each nonlinear operator $T$ with which we are dealing maps zero element to zero, i.e., satisfies $T 0=0$.

Definition 2.2. We say that a (nonlinear) operator $T$ satisfies the weak Hammerstein property if for arbitrary disjoint $u, v \in X$ and any $x \in X$ the following inequality

$$
|T(u+x+v)-T(u+x)| \leq|T(x+v)|+|T(x)|
$$

holds.

Letting $x=0$ in the above inequality we obtain $|T(u+v)-T(u)| \leq|T(v)|$, whence

$$
|T(u+v)| \leq|T(u)|+|T(v)|
$$

for arbitrary disjoint $u, v \in X$.

Let $T: X \rightarrow Y$ be an operator (possibly nonlinear), where $X$ and $Y$ are vector lattices which are represented on some compact spaces $K_{X}$ and $K_{Y}$, 
respectively. Moreover, as soon as some $e \in X_{+}$is fixed, we will always assume that $X$ is represented as $\{X, e\}$. Under these agreements we introduce the following open subset of $K_{Y}$ :

$$
U(T, e)=\bigcup\{\operatorname{cz}(T x): x \in X(e)\} .
$$

For each $t \in U(T, e)$ we denote by $\mathscr{S}(T, t, e)$ the collection of all those open subsets $G$ of $\operatorname{supp}(e)=e^{-1}(1)$ for which $(T x)(t)=0$ for each $x \in X(e)$ satisfying $\operatorname{cl}(\operatorname{cz}(x)) \subseteq G$. Finally, let

$$
\tau(t)=\operatorname{supp}(e) \backslash \bigcup\{G: G \in \mathscr{S}(T, t, e)\},
$$

that is, $\tau(\cdot)$ is a set-valued mapping from $U(T, e)$ into the collection of closed subsets of $K_{X}$.

The next lemma is an extension of Propositions 3.1 and 3.2 in [A], which were established there for linear operators.

Lemma 2.3. If $T$ satisfies the weak Hammerstein property, then the following statements are true.

(1) $\mathscr{S}(T, t, e)$ is an ideal of open subsets of $\operatorname{supp}(e)$.

(2) Each $\tau(t) \neq \varnothing$, and if for some $u, v \in X(e)$ we have $u[\tau(t)]=v$, then $(T u)(t)=(T v)(t)$. hold.

If, additionally, $T$ preserves disjointness, then also the next two properties

(3) For each $t \in \mathrm{cz}(T e)$ and for each $h_{1} \in\{e\}^{d d}$ satisfying $h_{1}(\tau(t)) \neq \infty$ the following implication is true: if $h_{2} \in X(e)$ and $h_{2}[\tau(t)]=h_{1}$, then $\left(T h_{1}\right)(t)=\left(T h_{2}\right)(t)$.

(4) For every point $t \in U(T, e)$, the set $\tau(t)$ is a singleton and the introduced mapping $\tau: U(T, e) \rightarrow \operatorname{supp}(e)$ is continuous.

Proof. (1) Let $h \in X(e)$ and $\operatorname{cl}(\operatorname{cz}(h)) \subseteq G_{1} \cup G_{2}$, where $G_{1}, G_{2} \in \mathscr{S}(T, t, e)$. Find disjoint open subsets $V_{1}, V_{2}$ of $\operatorname{supp}(e)$ such that $\operatorname{cl}(\operatorname{cz}(h)) \backslash G_{i} \subseteq V_{i} \subseteq$ $\operatorname{cl}\left(V_{i}\right) \subseteq G_{j}$, where $i, j=1,2, i \neq j$. There are $h_{1}, h_{2} \in X(e)$ satisfying $\left|h_{i}\right| \leq|h|, h_{i}\left[\operatorname{cl}(\operatorname{cz}(h)) \backslash G_{i}\right]=h$, and $h_{i}\left[\operatorname{supp}(e) \backslash V_{i}\right]=0$. Hence, $h_{1}$ and $h_{2}$ are disjoint, and consequently the weak Hammerstein property implies that

$$
\left|T h-T\left(h-h_{1}\right)\right| \leq\left|T\left(h-h_{2}\right)\right|+\left|T\left(h-h_{1}-h_{2}\right)\right| .
$$

Note that $\operatorname{cl}\left(\operatorname{cz}\left(h-h_{1}-h_{2}\right)\right) \subseteq G_{1} \cap G_{2}$ and $\operatorname{cl}\left(\operatorname{cz}\left(h-h_{i}\right)\right) \subseteq G_{i}$. Therefore, $T h(t)=0$, i.e., $G_{1} \cup G_{2} \in \mathscr{S}(T, t, e)$ establishing that $\mathscr{S}(T, t, e)$ is an ideal.

(2) Fix any $t \in U(T, e)$. There exists some $x \in X(e)$ such that $(T x)(t) \neq 0$, and therefore $\tau(t) \neq \varnothing$.

Notice that if $f[\tau(t)]=0$ for some $f \in X(e)$, then $(T f)(t)=0$ since $\operatorname{cl}(\operatorname{cz}(f)) \subseteq G \in \mathscr{S}(T, t, e)$. Let $u, v \in X(e)$ and $u[\tau(t)]=v$. We can find an element $h \in X(e)$ such that $h[\tau(t)]=u$ and $h[\operatorname{cl}(\operatorname{cz}(u-v))]=0$. Then $(u-h)[\tau(t)]=0,(v-h)[\tau(t)]=0$, and $(u \wedge v-h)[\tau(t)]=0$. Applying the weak Hammerstein property to the functions $u=(u-u \wedge v)+(u \wedge v-h)+h$ and $v=(v-u \wedge v)+(u \wedge v-h)+h$ we can conclude that $T u(t)=T(u \wedge v)(t)$ and $T v(t)=T(u \wedge v)(t)$, whence $T u(t)=T v(t)$.

(3) Take any $h \in X(e)$ satisfying the following two conditions $h[\tau(t)]=$ $h_{1}$ and $h\left[\mathrm{cl}\left(\mathrm{cz}\left(h_{1}-h_{2}\right)\right]=0\right.$. Our claim will be established if we show that 
$T h(t)=T h_{i}(t), i=1,2$. Let $G$ be an open neighborhood of $\tau(t)$ on which $h$ and $h_{i}$ coincide. There exists a function $f \in X(e)$ such that $f[\tau(t)]=h$ and $f[\operatorname{supp}(e) \backslash G]=0$. As $h_{i}=\left(h_{i}-h\right)+(h-f)+f, i=1,2$, the weak Hammerstein property implies that

$$
\left|T h_{i}-T h\right|(t) \leq\left|\left(T h_{i}-h\right)\right|(t)+|T(h-f)|(t)=\left|T\left(h_{i}-h\right)\right|(t) .
$$

We can find $e^{\prime} \in X(e)$ satisfying $e^{\prime}[\tau(t)]=e$ and $e^{\prime}\left[\operatorname{cl}\left(\operatorname{cz}\left(h_{i}-h\right)\right]=0\right.$. Then, by (2), $T e^{\prime}(t)=T e(t) \neq 0$ and $\left|T\left(h_{i}-h\right)\right|(t)=0$ since $\left|e^{\prime}\right| \wedge\left|h_{i}-h\right|=0$. Therefore, $T h_{i}(t)=T h(t)$ for $i=1,2$ and we are done.

(4)This can be verified similar to the case of linear operators in [A].

Let $T: X \rightarrow Y$ be a disjointness-preserving operator, and let $\tau: U(T, e) \rightarrow$ $\operatorname{supp}(e)$ be the mapping introduced right before the previous lemma. For each $x \in\{e\}^{d d}$ and each $t \in G(x):=U(T, e) \backslash \tau^{-1}\left(x^{-1}(\infty)\right)$ let

$$
(D x)(t)=(T x(\tau(t)) e)(t) .
$$

That is, $D x$ is a function defined on the set $G(x)$. Now, for each $(t, \lambda) \in$ $U(T, e) \times \mathbb{R}$ let $E(t, \lambda)=T(\lambda e)(t)$; in other words, we have defined an extended-valued function $E(t, \lambda): U(T, e) \times \mathbb{R} \rightarrow \mathbb{R} \cup\{ \pm \infty\}$. For each $t \in G(x)$ we have $(D x)(t)=E(t, x(\tau(t)))$, that is, on the set $G(x)$ the function $D x$ coincides with the value at $x$ of the composition operator induced by the function $E(t, \lambda)$ and the mapping $\tau$. Lemma 2.3 has established a close relationship between $D x$ and $T x$ which might suggest that $D x=T x$ on $U(T, e)$. However, as shown in Example 2 in [A], this is not true even when $T$ is a linear operator. Nevertheless, we shall show in Theorem 2.5 that under some natural conditions the above equality holds "almost everywhere" on $U(T, e)$. Therefore, the equality $D x=T x$ can be interpreted as a local representation of $T$ in the form of a composition operator.

A simple illustration is in order. Let $X=C([0,1]), Y=C(\beta(0,1])$, and $T: X \rightarrow Y$ be defined by

$$
(T x)(t)=\min \{|x(t)| / t, 1\}, \quad t \in(0,1] .
$$

Letting $e \equiv 1$, we have that in this case $U(T, e)=\beta(0,1]$, the Stone-Chech compactification of $(0,1]$, and the above representation holds on the set $(0,1]$ which is dense in $U(T, e)$.

Definition 2.4. We will say that an operator $T: X \rightarrow Y$ between vector lattices is quasi-linear if:

(1) for each $w \in X$ there exists a number $\lambda(w) \in \mathbb{R}_{+}$such that

$$
|T u-T v| \leq \lambda(w)|T(u-v)|,
$$

whenever $|u|,|v| \leq|w|$, and

(2) for each $u \in X$ and $r \in \mathbb{R}$ there exist real numbers $k(r, u)>0$ and $\ell(r, u)>0$ such that for each $v \in[-|u|,|u|]$

$$
\ell(r, u)|T v| \leq|T(r v)| \leq k(r, u)|T v|
$$

and also $\lim k(r, u)=0$ as $r \rightarrow 0$.

We denote by $D Q L H(X \rightarrow Y)$ the collection of all disjointness-preserving quasi-linear operators satisfying the weak Hammerstein property. 
Theorem 2.5. For each $T \in D Q L H(X \rightarrow Y)$ the following statements are equivalent.

(1) $T$ is $\left(r_{u}-r_{u}\right)$-continuous.

(2) $T$ is $\left(r_{u}-o\right)$-continuous.

(3) For each $e \in X_{+}$and each $x \in X(e)$ there is a meager subset $R(x) \subset$ $U(T, e)$ such that $T x=D x$ on $U(T, e) \backslash R(x)$.

Moreover, if $Y$ is a normed lattice, then each of the previous statements is equivalent to:

(4) $T$ is $\left(r_{u}-\|\cdot\|\right)$-continuous.

Proof. (2) $\Rightarrow(3)$. Let $f \in X(e)$, and let

$$
I(n, \ell):=f^{-1}\left[\frac{2 \ell}{2 n}, \frac{2 \ell+1}{2 n}\right] \text { and } J(n, \ell):=f^{-1}\left[\frac{2 \ell+1}{2 n}, \frac{2 \ell+2}{2 n}\right] \text {, }
$$

for all $n, \ell \in N$. Find $a_{n} \in X(e)$ satisfying $\left|a_{n}-f\right| \leq \frac{1}{n} e, a_{n}[I(n, \ell)]=$ $\frac{2 l+1}{2 n} e$, and similarly, $b_{n} \in X(e)$ satisfying $\left|b_{n}-f\right| \leq \frac{1}{n} e, b_{n}[J(n, \ell)]=\frac{2 l+2}{2 n} e$. By (2) there is a meager set $R \subset U(T, e)$ such that $T a_{n}(t) \rightarrow T f(t)$ and $T b_{n}(t) \rightarrow T f(t)$ for each $t \in U(T, e) \backslash R$.

For each such $t$ let

$$
N_{1}(t)=\left\{n \in \mathbb{N}: \tau(t) \in \bigcup_{\ell} I(n, \ell)\right\}, \quad N_{2}(t)=\left\{n \in \mathbb{N}: \tau(t) \in \bigcup_{\ell} J(n, \ell)\right\} .
$$

Suppose $N_{1}(t)$ is infinite. For $n \in N_{1}$ there exists $\ell(n) \in \mathbb{N}$ such that $\tau(t) \in$ $I(n, \ell(n))$. Hence, $T a_{n}(t)=T\left(\frac{2 l(n)+1}{2 n} e\right)(t)$ for $n \in N_{1}$. Since $f \in X(e)$, there is $m \in \mathbb{N}$ satisfying $|f| \leq m e$. Let $\lambda=\lambda(m e)$ be a constant guaranteed by Definition 2.4(1). Then we have

$$
\begin{aligned}
& \left|T\left(\frac{2 \ell(n)+1}{2 n} e\right)-T(f(\tau(t)) e)\right|(t) \\
& \quad \leq \lambda k\left(\frac{2 \ell(n)+1}{2 n}-f(\tau(t), e)\right) \cdot|T e|(t) \rightarrow 0 .
\end{aligned}
$$

So $T f(t)=D f(t)$.

(3) $\Rightarrow(1)$. Fix an $n \in \mathbb{N}$, and find $\delta>0$ such that $k(r, e)<\frac{1}{n}$ whenever $|r|<\delta$. If $|f-h| \leq \delta e$, then by (3) there exists a dense subset $M$ of $U(T, e)$ on which $|T f-T h|(t)=|D f-D h|(t) \leq \lambda \frac{1}{n}|T e|(t)$. This implies that $\mid T f-$ $T h\left|\leq \lambda \frac{1}{n}\right| T e \mid$, that is, $T$ is $\left(r_{u}-r_{u}\right)$-continuous.

(3) $\Rightarrow$ (4) can be proved similar to the proof of the previous implication.

$(4) \Rightarrow(3)$. For $f \in X(e)$ let $a_{n}, b_{n}$ be the elements constructed above in our proof of implication (2) $\Rightarrow(3)$. Then, letting $u_{n}=\left|T\left(a_{n}-f\right)\right|+\left|T\left(b_{n}-f\right)\right|$, we have $\left\|u_{n}\right\| \rightarrow 0$. Therefore, $\bigwedge_{n \geq k}\left|u_{n}\right|=0$ for each $k \in \mathbb{N}$, and there is a meager set $R \subset U(T, e)$ such that for each $t \in U(T, e) \backslash R$ we can find a subsequence $n(k)$ for which $u_{n(k)}(t) \rightarrow 0$. In particular, we have

$$
\left|T a_{n(k)}-T f\right|(t) \leq \lambda\left|T\left(a_{n(k)}-f\right)\right|(t) \leq \lambda u_{n(k)}(t)
$$

and hence, $T a_{n(k)}(t) \rightarrow T f(t)$. Repeating the arguments from (2) $\Rightarrow(3)$, we obtain that $T a_{n(k(s))}(t) \rightarrow D f(t)$ and $T b_{n(k(s))}(t) \rightarrow D f(t)$, whence $T f(t)=$ $D f(t)$. 
Theorem 2.6. If $T \in D Q L H(X \rightarrow Y)$ is $\left(r_{u}-r_{u}\right)$-continuous, then $T$ satisfies the Hammerstein property. Moreover, $T$ is additive if and only if $T$ is homogeneous.

Proof. To verify that $T$ satisfies the Hammerstein property fix two arbitrary disjoint $u, v \in X$ and any $x \in X$ (see Definition 2.1). Fix also any $e \in X_{+}$ satisfying $u, v, x \in X(e)$. We denote by $E$ the set $\{u+v+x, u+x, v+x$, $x\}$. By Theorem 2.5 there exists a dense $M \subset U(T, e)$ such that on $M$ we have $T h=D h$ for each $h \in E$. Fix $t \in M$. We may assume that $u(\tau(t))=0$. Then

$$
T(u+v+x)(t)=D(u+v+x)(t)=D(v+x)(t)=T(v+x)(t)
$$

and similarly

$$
T(x)(t)=D(x)(t)=D(u+x)(t)=T(u+x)(t) .
$$

This means that $T$ satisfies the Hammerstein property.

It is well known that any additive $\left(r_{u}-r_{u}\right)$-continuous operator is homogeneous. Therefore, to finish the proof it remains to verify the converse statement, that if $T$ is homogeneous (and $\left(r_{u}-r_{u}\right)$-continuous), then $T$ is additive. Take arbitrary $a, b \in X(e)$, and let $A=\{a+b, a, b\}$. Using Theorem 2.5 again we can find a dense $M \subset U(T, e)$ such that on $M$ we have $T h=D h$ for each $h \in A$. Note that $D(\lambda e)(t)=T(\lambda e(\tau(t)) e)(t)=D((\lambda e)(\tau(t)) e)(t)$ for an arbitrary scalar $\lambda$, whence

$$
\begin{aligned}
T(a+b)(t) & =D(a+b)(t)=T((a+b) \tau(t) e)(t)=(a+b)(\tau(t)) \cdot T e(t), \\
T(a)(t) & =D(a)(t)=D(a(\tau(t)) e)(t)=T(a(\tau(t)) e)(t)=a(\tau(t)) \cdot T e(t), \\
T(b)(t) & =D(b)(t)=D(b(\tau(t)) e)(t)=T(b(\tau(t)) e)(t)=b(\tau(t)) \cdot T e(t) .
\end{aligned}
$$

The above three identities imply that $T(a+b)=T a+T b$.

\section{DISJOINTNESS PRESERVING ISOMORPHISMS}

Definition 3.1. We say that an operator $T: X \rightarrow Y$ from a vector lattice $X$ into a normed lattice $Y$ satisfies the condition $(D N B)$, if $T$ sends order bounded $d$-sequences into norm bounded; i.e., for each order bounded sequence $\left\{x_{n}\right\}$ in $X$ consisting of pairwise disjoint elements the scalar sequence $\left\{\left\|T x_{n}\right\|\right\}$ is bounded.

Lemma 3.2. Let $T \in D Q L H(X \rightarrow Y)$, where $Y$ is a normed lattice.

(1) If $X$ is uniformly complete, then $T$ satisfies $(D N B)$.

(2) If $T$ satisfies $(D N B)$, then for each $e \in X_{+}$there exists $m=m(e) \in \mathbb{N}$ such that the following set is finite:

$$
\begin{aligned}
E(m, e)=\{s \in \operatorname{supp}(e): & (\forall G(s))(\exists x \in X):|x| \leq e, \\
& x[\operatorname{supp}(e) \backslash G(s)]=0,\|T x\| \geq m\} .
\end{aligned}
$$

Proof. (1) Let $\left\{f_{n}\right\}$ be a disjoint sequence in $X$ such that $\left|f_{n}\right| \leq e \in X$. Assume, contrary to what we claim, that $\sup \left\|T f_{n}\right\|=\infty$. Without loss of generality we can suppose that $\sup \left\|T f_{n}\right\| \geq n$. Next find an increasing sequence $\{n(k)\} \subset \mathbb{N}$ such that $n(k)^{-1 / 2} \leq \ell\left(\frac{1}{k}\right)$. The uniform completeness of $X$ implies that $h=\sum \frac{1}{k} f_{n(k)}$ exists in $X$. Consequently $\|T h\| \geq \| T\left(\left(\frac{1}{k} f_{n(k)} \| \geq\right.\right.$ $n(k)^{1 / 2}$, a contradiction. 
(2) If each $E(n, e)$ was infinite, then there would exist some $s \in \operatorname{supp}(e)$ such that for each neighborhood $G(s)$ of $s$ and for each $n \in \mathbb{N}$ the intersection $(G(s) \backslash\{s\}) \cap E(m, e) \neq \varnothing$ for some $m \geq n$. This immediately implies the existence of an order-bounded disjoint sequence on which $T$ is not norm bounded, a contradiction.

Lemma 3.3. Let $T \in D Q L H(X \rightarrow Y)$.

(1) For each $n \in \mathbb{N}$ and each $x \in X(e)$ there is a meager set $R(x, n) \subset$ $U(T, e)$ such that $T x=D x$ on $\tau^{-1}(\operatorname{supp}(e) \backslash E(n, e)) \backslash R(x, n)$.

(2) If $s$ is a discrete point in $\operatorname{supp}(e)$, then $T x=D x$ on $\tau^{-1}(s)$.

(3) If $t \in U(T, e) \cap \mathrm{cz}(T x)$ and $T x(t)=D x(t)$, then $T e(t) \neq 0$.

(4) Let $T$ be $\left(r_{u}-r_{u}\right)$-continuous on $\{e\}^{d d}$. Then for each $x \in\{e\}^{d d}$ the equality $T x=D x$ holds on the set $\tau^{-1}\left(\operatorname{supp}(e) \backslash x^{-1}(\infty)\right) \backslash P(x)$ for some meager set $P(x)$.

Proof. (1) If $s \in \operatorname{supp}(e) \backslash E(n, e)$, then there is a neighborhood $G(s)$ with the following property:

(*) $\quad$ if $h \in X,|h| \leq e$, and $\operatorname{cl}(\operatorname{cz}(h)) \subset G(s)$, then $\|T h\| \leq n$

Construct now a complete family of disjoint open sets

$$
W_{\alpha} \subset \tau^{-1}(\operatorname{supp}(e) \backslash E(n, e))
$$

such that $\operatorname{cl}\left(\tau\left(W_{\alpha}\right) \subset G_{\alpha}\right.$, where $G_{\alpha} \subset \operatorname{supp}(e)$ are open and satisfy $(*)$.

Let $f \in X(e)$. For each $\alpha$ find an element $g_{\alpha} \in X(e)$ such that $g_{\alpha}\left[\operatorname{cl}\left(\tau\left(W_{\alpha}\right)\right)\right]$ $=f$ and $g_{\alpha}\left[\operatorname{supp}(e) \backslash G_{a}\right]=0$. Now imitating the proof of the implication $(4) \Rightarrow(3)$ in Theorem 2.5 we can find a meager set $R(f, n, \alpha)$ satisfying $T g_{\alpha}=D g_{\alpha}$ on $U(T, e) \backslash R(f, n, \alpha)$. However, by Lemma 2.3, $T g_{\alpha}=T f$ and $D g_{\alpha}=D f$ on $W_{\alpha}$. So $T f=D f$ on $W_{\alpha} \backslash R(f, n, \alpha)$. This allows us to choose the required set $R(f, n)$.

(2) This follows directly from Lemma 2.3 .

(3) Notice that

$$
0 \neq|T h|(t)=|T h(\tau(t)) e|(t) \leq k(h(\tau(t)))|T(e)|(t),
$$

where $k(h(\tau(t)))$ is a constant guaranteed by Definition $2.4(2)$. This clearly implies statement (3).

(4) The validity of this statement follows from Theorem 2.5, Lemma 2.3, and the arguments in (1).

Recall [AVK2] that an operator $T: X \rightarrow Y$ between two vector lattices is said to be a $d$-isomorphism if

$$
u, v \in X \text { are disjoint in } X \text { iff } T u, T v \text { are disjoint in } Y \text {. }
$$

Clearly for each d-isomorphism $T$ its kernel $T^{-1}(0)=\{0\}$. Consequently, if $T$ is a linear d-isomorphism, then $T$ is one-to-one. The next two theorems are the main results of this section. The first of them shows that under some "minimal" continuity conditions the operators from $D Q L H(X \rightarrow Y)$ are d-isomorphisms, and the second shows that these continuity conditions are automatically verified if $X$ is a Banach lattice and $T$ satisfies some simple algebraic conditions. 
Theorem 3.4. Let $T \in D Q L H(X \rightarrow Y)$ satisfy $T^{-1}(0)=\{0\}$, and let $Y$ be a normed lattice. Then either of the following two conditions:

(1) $T$ is $\left(r_{u}-r_{u}\right)$-continuous,

(2) $T$ is $(D N B)$

implies that $T$ is a d-isomorphism. Furthermore, if $T X$ is order dense in $Y$, then (2) implies (1).

Proof. To prove that $T$ is a d-isomorphism, we will suppose that $g_{1}, g_{2} \in X(e)$ are not disjoint and then show that $T g_{1}, T g_{2}$ are not disjoint in $Y$. Since $T^{-1}(0)=\{0\}$, we can conclude that $\tau^{-1}\left(\mathrm{cz}\left(g_{1}\right) \cap \mathrm{cz}\left(g_{2}\right)\right) \neq \varnothing$. We need a point $t \in \tau^{-1}\left(\mathrm{cz}\left(g_{1}\right) \cap \mathrm{cz}\left(g_{2}\right)\right)$ for which $\left(T g_{i}\right)(t)=\left(D g_{i}\right)(t), i=1,2$. Then by Lemma 3.3, $\left|T g_{i}\right|(t) \geq \ell\left(g_{i}(\tau(t)), e\right) \cdot|T(e)|(t) \neq 0$, that is, $t \in \operatorname{cz}\left(T g_{1}\right) \cap$ $\mathrm{cz}\left(T g_{2}\right)$.

In case (1) a required point exists by Theorem 2.5. In case (2), by Lemma 3.2 the set $E(m, e)$ is finite for some $m \in \mathbb{N}$. If $\operatorname{cz}\left(g_{1}\right) \cap \mathrm{cz}\left(g_{2}\right) \backslash E(m, e) \neq \varnothing$, then the condition $T^{-1}(0)=\{0\}$ and Lemma 3.3 imply the existence of a required point.

If $\mathrm{cz}\left(g_{1}\right) \cap \mathrm{cz}\left(g_{2}\right) \backslash E(m, e)=\varnothing$, then there is a point $p \in E(m, e)$ which is discrete in $\operatorname{supp}(e)$, and once again the condition that $T^{-1}(0)=\{0\}$ and Lemma 3.3 imply that any $t \in \tau^{-1}(p)$ is a required point.

It remains to verify that if $T X$ is order dense in $Y$, then (2) implies (1). Assume first that the set $E(m, e)$ is finite. If for any nondiscrete point $t \in$ $E(m, e)$ the set int $\left(\tau^{-1}(t)\right)$ is empty, then Lemma 3.3 implies that for each $f \in$ $X(e)$ we can find a meager set $R(f)$ such that $T f=D f$ on $U(T, e) \backslash R(f)$. Therefore, $T$ is $\left(r_{u}-r_{u}\right)$-continuous by Theorem 2.5 .

Assume now that $\operatorname{int}\left(\tau^{-1}(t)\right) \neq \varnothing$. Pick a function $f \in X$ such that $T f \neq 0$ and $\operatorname{cz}(T f) \subseteq \operatorname{int}\left(\tau^{-1}(t)\right)$. Notice that $|f| \wedge e \neq 0$ (otherwise, $T f \equiv 0$ on $U(T, e))$ and there exists some $h \in X(e)$ such that the intersection $\operatorname{cz}(T h) \cap$ $\operatorname{cz}(T f) \neq \varnothing$. Hence, $f$ and $h$ are not disjoint. Now find $g \in X(e)$ such that $g$ and $f$ are not disjoint and $g[t]=0$. By the first part of the present theorem $T$ is a d-isomorphism, and consequently the elements $T g$ and $T f$ in $Y$ are not disjoint either. However, this is impossible since $T g \equiv 0$ on $\tau^{-1}(t)$ and $\operatorname{cz}(T f) \subseteq \operatorname{int}\left(\tau^{-1}(t)\right)$.

Theorem 3.5. Let $X$ be a uniformly complete vector lattice and $Y$ be a normed lattice. Let $T \in D Q L H(X \rightarrow Y)$ be a one-to-one and onto operator satisfying the following property.

$(\beta)$ For each $x \in X$ and for each $0<r \in \mathbb{R}$ there exists a positive number $d(x, r)$ such that $\left|T\left(r_{1} x\right)-T\left(r_{2} x\right)\right| \geq d(x, r)|T(x)|$ whenever $\left|r_{1}-r_{2}\right| \geq r$.

Then both $T$ and $T^{-1}$ are $\left(r_{u}-r_{u}\right)$-continuous. In particular, if $X$ and $Y$ are Banach lattices, then $T$ and $T^{-1}$ are continuous.

Proof. Since $X$ is uniformly complete, Lemma 3.2 implies that $T$ satisfies (DNB), and consequently, by Theorem 3.4 , the operator $T$ is a $\left(r_{u}-r_{u}\right)$ continuous d-isomorphism. Observe that if $|T h| \leq|T g|$ for some $h, g \in X$, then $h \in\{g\}^{d d}$. Indeed, otherwise, we can find $f \in X$ such that $|h| \wedge|f| \neq 0$ and $|h| \wedge|g|=|f| \wedge|g|=0$.

Now assume that $\left|T h_{1}\right|,\left|T h_{2}\right| \leq|T g|$ and $\left|T h_{1}-T h_{2}\right| \leq \frac{1}{2} d\left(g, \frac{1}{n}\right)|T(g)|$. We claim that $\left|h_{1}-h_{2}\right| \leq \frac{1}{n} e$, where $e=|g|$. First check that for any $f \in\{e\}^{d d}$ the set $E=\operatorname{int}\left(\tau^{-1}\left(f^{-1}(\infty)\right)\right)$ is empty. Otherwise, we will be able to construct 
a sequence $f_{n} \in X(e)$ such that $\left|f_{n}\right| \leq \frac{1}{n}|f|$ and $f_{n}\left[f^{-1}(\infty)\right]=e$. Then the sequence $\left\{T f_{n}\right\}$ is $r_{u}$-convergent to 0 in $Y$ and $T f_{n}=T e$ on $E$. So $T e=0$ on $E$. Take any $h \in X(e)$ such that $\operatorname{cz}(T h) \subseteq E$. Since $T$ is $\left(r_{u}-r_{u}\right)$ continuous, there is some point $t \in \mathrm{cz}(T h)$ at which $T h(t)=D h(t)$. By Lemma 3.3, $T e(t) \neq 0$ contrary to the fact that $t \in E$. Hence, indeed, $E=\varnothing$.

Now suppose that there is a nonempty open set $G$ in $\operatorname{supp}(e)$ such that $\left|h_{1}-h_{2}\right| \geq \frac{1}{n} e$ on $G$. Since $\operatorname{int}\left(\tau^{-1}\left(h_{i}^{-1}(\infty)\right)\right)=\varnothing, i=1,2$, and in view of Lemma 3.3 there is a point $t \in \tau^{-1}(G)$ such that $T h_{i}(t)=D h_{i}(t)$. Since $\left|h_{1}(\tau(t))-h_{2}(\tau(t))\right| \geq \frac{1}{n}$, either $h_{1}(\tau(t)) \neq 0$ or $h_{2}(\tau(t)) \neq 0$ and, consequently, either $T h_{1}(\tau(t)) \neq 0$ or $T h_{2}(\tau(t)) \neq 0$. By Lemma 3.3, Te $(t) \neq 0$.

Suppose that $g(\tau(t))<0$. Then

$$
\begin{aligned}
\frac{1}{2} d\left(g, \frac{1}{n}\right)|T g|(t) & \geq\left|T h_{1}-T h_{2}\right|(t)=\left|T\left(h_{1}(\tau(t)) e\right)-T\left(h_{2}(\tau(t)) e\right)\right|(t) \\
& =\left|T\left(-h_{1}(\tau(t)) g\right)-T\left(-h_{2}(\tau(t)) g\right)\right|(t) \geq d\left(g, \frac{1}{n}\right)|T g|(t),
\end{aligned}
$$

whence $|T g|(t)=0 \quad(*)$.

Our next step is to show that there exists a constant $\gamma>0$ such that $|T g| \geq$ $\gamma|T e|$. As $(T e)(t) \neq 0$ this implies immediately that $|T g|(t) \neq 0$, contrary to $(*)$.

Since $g_{+}, g_{-}$are disjoint element and $T$ preserves disjointness, the elements $T g_{+}, T g_{-}$are also disjoint and hence,

$$
\left|T g_{+}\right|+\left|T g_{-}\right|=\left|T g_{+}+T g_{-}\right|=\left|T g_{+}-T g_{-}\right| \text {. }
$$

This and the fact that $T$ satisfies the weak Hammerstein property imply that

$$
\left|T\left(g_{+}+g_{-}\right)\right| \leq\left|T g_{+}\right|+\left|T g_{-}\right|=\left|T g_{+}-T g_{-}\right| \text {. }
$$

Now we are going to use that $T$ is a quasi-linear operator (see Definition 2.4). We have

$$
|T g|=\left|T\left(g_{+}-g_{-}\right)\right| \geq \frac{1}{\lambda(|g|)}\left|T\left(g_{+}\right)-T\left(g_{-}\right)\right|,
$$

and hence, in view of $(* *)$,

$$
|T g| \geq \frac{1}{\lambda(|g|)}\left|T\left(g_{+}+g_{-}\right)=\right| \frac{1}{\lambda(|g|)}|T e| .
$$

That is, $\gamma=1 / \lambda(|g|)$ is the desired constant.

Thus, we have proved that $\left|h_{1}-h_{2}\right| \leq \frac{1}{n} e$, that is, $T^{-1}$ is $\left(r_{u}-r_{u}\right)$-continuous.

Remark. Using arguments similar to those in Lemma 3.4 and Theorems 3.4 and 3.5 we can obtain analogous results without assuming that $Y$ is a normed lattice. For example,

If $T \in D Q L H(X \rightarrow Y)$ is an $\left(r_{u}-r_{u}\right)$-continuous operator from a vector lattice $X$ into a vector lattice $Y$, then $T$ is a d-isomorphism if and only if $T^{-1}(0)=\{0\}$.

To prove this, we (as in Theorem 3.4) take $t \in \tau^{-1}\left(\mathrm{cz}\left(g_{1}\right) \cap \mathrm{cz}\left(g_{2}\right)\right)$ at which $\left(T g_{1}\right)(t)=\left(D g_{1}\right)(t)$. Then $|T e|(t) \neq 0$, and consequently Theorem 2.5 implies that $\left|T g_{i}\right|(t) \neq 0$. 
We refer to $[\mathrm{K}]$ for some additional results on automatic $\left(r_{u}-r_{u}\right)$-continuity of nonlinear operators satisfying (DNB).

It is obvious that each linear operator satisfies the condition $(\beta)$ of the previous theorem. Therefore, by that theorem, each linear disjointness-preserving one-to-one operator from a uniformly complete vector lattice onto a normed lattice is $\left(r_{u}-r_{u}\right)$-continuous and hence (by Theorem 3.4), is a d-isomorphism. In fact, the surjectivity of the operator is not essential (see below). Also more can be said about the automatic $\left(r_{u}-r_{u}\right)$-continuity of d-isomorphisms. All this is done in the next theorem, which solves in the affirmative both questions raised by Y. Abramovich in Problem 1 [HP, p. 143]. We reiterate once more that the significance of the concluding part of Theorem 3.6 lies in the fact that an algebraic condition of being a d-isomorphism is equivalent to a topological condition of being continuous.

Theorem 3.6. Let $T$ be a one-to-one linear disjointness-preserving operator from a uniformly complete vector lattice $X$ into a normed lattice $Y$. Then $Y$ is a d-isomorphism.

Moreover, if the image $T X$ is order dense in $Y$ (in particular, if $T X=Y$ ), then $T$ is a d-isomorphism if and only if $T$ is $\left(r_{u}-r_{u}\right)$-continuous.

Proof. By Lemma 3.2 the operator $T$ satisfies (DNB) and hence, is a d-isomorphism by Theorem 3.4 .

We turn now to the second statement. If $T$ is $\left(r_{u}-r_{u}\right)$-continuous, then, again by Theorem 3.4, the operator $T$ is a d-isomorphism. It remains to prove the converse statement that if $T$ is a d-isomorphism, then $T$ is $\left(r_{u}-r_{u}\right)$ continuous. Only in proving this will we use the extra assumption that the image $T X$ is order dense in $Y$. To prove our claim it is enough to show that the image $T([0, e])$ is order bounded in $Y$ for each $e \in X_{+}$. Without loss of generality we can assume that $X=X(e)$.

Observe that $T x \in\{T e\}^{d d}$ for each $x \in[0, e]$. Indeed, otherwise using our assumption that $T X$ is order dense in $Y$ we can find $x^{\prime} \in[0, e]$ such that $\left|T x^{\prime}\right| \wedge|T x| \neq 0$ while $\left|T x^{\prime}\right| \wedge|T e|=0$, contradicting the condition that $T$ is a d-isomorphism.

By Lemma 3.2 there exists some $m \in \mathbb{N}$ such that the set $E(m, e)$ introduced in Lemma 3.2 is finite. We will show that for each nondiscrete point $t \in E(m, e)$ the set $\operatorname{int}\left(\tau^{-1}(t)\right)$ is empty. If not, then (since $T X$ is order dense in $Y$ ) there exists some function $0 \neq f \in X$ such that $\operatorname{cz}(T f) \subset \operatorname{int}\left(\tau^{-1}(t)\right)$. Find further an element $g \in X(e)=X$ such that $|g| \wedge|f| \neq 0$ and $g[t]=0$. By Lemma 2.3, $T g \equiv 0$ on $\left.\tau^{-1}(t)\right)$. This implies that $|T g| \wedge|T f|=0$, contrary to the fact that $T$ is a d-isomorphism.

Finally, by Lemma 3.3(4) for each $x \in X(e)$ there exists a meager set $P(x)$ such that $T x=D x$ on $\tau^{-1}(\operatorname{supp}(e)) \backslash(E(m, e) \cup P(x))$. In other words, on $\operatorname{cz}(T e)$ we have that $(T x)(t)=x(\tau(t)) \cdot(T e)(t)$. This means that $|T x| \leq|T e|$ for each $x \in[0, e]$, and the proof is finished.

Corollary 3.7. Let $T$ be a one-to-one linear disjointness-preserving operator from a Banach lattice $X$ onto a Banach lattice $Y$. Then $T$ is a continuous $d$ isomorphism.

The next two examples show that without assuming some type of completeness of the vector lattice $X$ or without assuming that $Y$ is a normed lattice, 
one cannot guarantee that the inverse of a one-to-one disjointness-preserving operator is also a disjointness-preserving operator.

Example 3.8. Let $X$ consist of all continuous piecewise linear functions on $[0,1]$, i.e., for each $x \in X$ there are a partition (depending on $x) 0=t_{1}<$ $t_{2}<\cdots<t_{n}=1$ of $[0,1]$ and numbers $a_{i}=a_{i}(x), b_{i}=b_{i}(x) \in \mathbb{R}$ such that $x(t)=a_{i} t+b_{i}$ for all $t \in\left[t_{i}, t_{i+1}\right]$, where $i=1,2, \ldots, n-1$. Let $\left\{q_{n}\right\}$ be a sequence of all rational numbers from $[0,1)$. Hence for each $x \in X$ and for each $n \in \mathbb{N}$ there exists a unique $i_{n}=i(x, n) \in \mathbb{N}$ such that $q_{n} \in\left[t_{i_{n}}, t_{i_{n}+1}\right)$. Finally, we denote by $\alpha \mathbb{N}$ the one-point compactification of the set $\mathbb{N}$ of all natural numbers, and let $Y=C(\alpha \mathbb{N})$.

Now we are in a position to define an operator $T: X \rightarrow Y$. For each $x \in X$ and each $k \in \mathbb{N}$ we let

$$
(T x)(k)= \begin{cases}\frac{1}{n} a_{i_{n}}(x) & \text { if } k=2 n \\ \frac{1}{n} b_{i_{n}}(x) & \text { if } k=2 n+1\end{cases}
$$

It is easy to see that $T$ is a one-to-one, linear, disjointness-preserving operator. If we take now $u=u(t)=1$ and $v=v(t)=t$, then $u, v \in X$, their images $T u$ and $T v$ are disjoint in $Y$, but $u, v$ are not disjoint.

Example 3.9. Let $X=C(Q)$ be a Dedekind completion of $C[0,1]$ represented on its (extremally disconnected compact) Stonean space $Q$. For each $q \in Q$ we denote by $I(q)$ the ideal in $X$ consisting of all functions vanishing on a neighborhood (depending on function) of $q$, that is,

$$
I(q)=\{f \in C(Q): f[q]=0\},
$$

and we denote by $\pi^{q}$ the canonical quotient mapping $\pi^{q}: C(Q) \rightarrow C(Q) / I(q)$. Let $\Psi(q)=\left\{\pi^{q}\left(h_{\alpha}^{q}\right): \alpha \in A(q)\right\}$ be an arbitrary Hamel basis in $C(Q) / I(q)$ containing $\pi^{q}(u)$ and $\pi^{q}(v)$, where $u$ and $v$ are the functions introduced in Example 3.8. Let $D(q)=\left\{t_{\alpha}^{q}: \alpha \in A(q)\right\}$ be a discrete space, and let

$$
D=\bigoplus\{D(q): q \in Q\}
$$

be the topological direct sum of spaces $D(q)$. As usual, $Y=C(D)$ denotes the space of all continuous functions on $D$.

For each $f \in C(Q)$ and each $q \in Q$ there is a unique representation

$$
f=\sum a\left(f, q, \alpha_{i}\right) h_{\alpha_{i}}^{q}+g
$$

where $a\left(f, q, \alpha_{i}\right) \in \mathbb{R}$ and $g \in I(q)$. We define an operator $T: X \rightarrow Y$ by letting $T(f)\left(t_{\alpha}^{q}\right)=a(f, q, \alpha) \quad(f \in X)$. Clearly $T$ is a linear operator. We claim that $T$ is one-to-one. Assume that $f \neq 0$. Then there exists a point $q \in Q$ such that $f \notin I(q)$. Hence we can find $\alpha \in A(q)$ satisfying $a(f, q, \alpha) \neq 0$, that is, $T f \neq 0$.

We claim that operator $T$ preserves disjointness. Indeed, if $f_{1}, f_{2}$ are disjoint functions in $X$, then for any $q \in Q$ either $f_{1}[q]=0$ or $f_{2}[q]=0$. Suppose $f_{1}[q]=0$. Then $a\left(f_{1}, q, \alpha\right)=0$ for any $\alpha \in A(q)$.

However, $T$ is not a d-isomorphism since $T(u) \wedge T(v)=0$. Indeed, for each $q \in Q$ we have that $\pi^{q}(u)=\pi^{q}\left(h_{\alpha(q)}^{q}\right)$ for some index $\alpha(q) \in A(q)$. Let $t_{\alpha}^{q} \in D$. If $\alpha=\alpha(q)$, then $T(v)\left(t_{\alpha}^{q}\right)=0$. If $\alpha \neq \alpha(q)$, then $T(u)\left(t_{\alpha}^{q}\right)=0$. 
The operator $T$ constructed above is useful in other situations as well. Let an operator $S: C(Q) \rightarrow C(Q) \oplus C(D)$ be defined by $S f=f \oplus T(f)$ for $f \in C(Q)$. Then $S$ is a linear d-isomorphism satisfying (DNB) (since $C(Q)$ is uniformly complete) but failing to be $\left(r_{u}-r_{u}\right)$-continuous. This shows that an extra assumption in Theorem 3.6 about the image of the operator is essential.

Similarly, fix any $t=t_{\alpha}^{q} \in D$ and let $\varphi(f)=(T f)(t)$. Then $\varphi$ is a linear disjointness-preserving (surjective) functional satisfying (DNB) but failing to be $\left(r_{u}-r_{u}\right)$-continuous. Of course, the functional $\varphi$ is not one-to-one, and that is why this example does not contradict Theorem 3.6.

Several comments are in order in connection with the Hammerstein property introduced in Definition 2.1. This property goes back to the theory of integral operators $[\mathrm{Ko}, \mathrm{Kr}]$ and this explains its importance. Recall that as we noticed after Definition 2.1 each operator with the Hammerstein property is disjointly additive. The latter property was introduced by Pinsker [P] as early as in 1938 (see also [DO]). Here we want to point out that there is a principal distinction between the operators with Hammerstein property and disjointly additive operators. We will illustrate this distinction with an example.

Let $T: C[0,1] \rightarrow \mathbb{R}$ be the following functional: $T x:=\min \{|x(t)|: t \in$ $[0,1]\}$. This functional is disjointly additive, $\left(r_{u}-r_{u}\right)$-continuous, and preserves disjointness. However, $T$ does not satisfy the Hammerstein property, and therefore, $T$ allows neither any natural integral representation nor a representation as a composition operator. The important Lemma 2.3 fails for this operator. In other words, the operators with the Hammerstein property (rather than the disjointly additive operators) may be viewed as a natural generalization of linear operators on vector lattices.

In conclusion I would like to express my thanks to Y. Abramovich for pointing out that C. Huijsmans and B. de Pagter [HP2] have also solved the problem posed in [HL] and constructed an example similar to Example 3.8.

\section{REFERENCES}

[A] Y. A. Abramovich, Multiplicative representation of operators preserving disjointness, Nederl. Akad. Wetensch. Proc. Ser. A 86 (1983), 265-279.

[AAK] Y. A. Abramovich, E. L. Arenson, and A. K. Kitover, Banach $C(K)$-modules and operators preserving disjointness, Pitman Res. Notes Math. Ser., vol. 277, Longman Sci. Tech., Harlow, 1992.

[AVK1] Y. A. Abramovich, A. I. Veksler, and A. V. Koldunov, Operators preserving disjointness, their continuity and multiplicative representation, Linear Operators and their Appl., Sbornik Nauchn. Trudov, Leningrad, 1981, pp. 13-34.

[AVK2] __ Operators preserving disjointness, Dokl. Akad. Nauk USSR 248 (1979), 1033-1036.

[AB] C. D. Aliprantis and O. Burkinshaw, Positive operators, Academic Press, New York, 1985.

[Art] W. Arendt, Spectral properties of Lamperti operators, Indiana Univ. Math. J. 32 (1983), 199-215.

[AH] W. Arendt and D. R. Hart, The spectrum of quasi-invertible disjointness preserving operators, J. Funct. Anal. 68 (1986), 149-167.

[DO] L. Drewnowski and W. Orlicz, On representation of orthogonally additive functionals, Bull. Acad. Polon. Sci. Ser Math. Astr. Phys. 17 (1969), 167-173.

[Har] D. R. Hart, Some properties of disjointness preserving operators, Indag. Math. (N.S.) 47 (1985), 183-197.

[HL] C. B. Huijsmans and W. A. J. Luxemburg, eds., Positive operators and semigroups on Banach lattices, Kluwer, Dordrecht, 1992. 
[HP1] C. B. Huijsmans and B. de Pagter, Disjointness preserving and diffuse operators, Compositio Math. 79 (1991), 351-374.

[HP2] Invertible disjointness preserving operators, Proc. Edinburgh Math. Soc. (2) 37 (1993), 125-132.

[Kit1] A. K. Kitover, Disjoint operator in Banach lattices, Dokl. Akad. Nauk USSR 250 (1980), 800-803.

[Kit2] _- Operators preserving disjointness and the mappings of extremally disconnected compact spaces, Optimizatsiya 40 (1987), 138-149.

[K] A. V. Koldunov, Automatic continuity of operators in vector lattices, Mat. Zametki 50 (1991), 67-73.

[Ko] I. I. Kolodner, Equations of Hammerstein in Hilbert spaces, J. Math. Mech. 13 (1964), 701-750.

[Kr] M. A. Krasnoselskii, Topological methods for non-linear integral equations, Pergamon Press, New York, 1964.

[P] A. G. Pinsker, Analytical representation of some disjointly-additive functionals, Dokl. Akad. Nauk USSR 18 (1938), 397-401.

[V] B. Z. Vulikh, Introduction to the theory of partially ordered spaces, Walters-Noordhoff, Groningen, 1967.

[W] A. W. Wickstead, Representation and duality of multiplication operators on Archimedean Riesz spaces, Compositio Math. 35 (1977), 225-238.

Department of Mathematics, The Russian Pedagogical University, 191186 St. PetersBURG, RUSSIA 